\title{
Model Helicopter Control Using Body-Mounted Vibro-Tactile Transducers
}

\author{
Rui Zhou1, Merhran Mehrandezh², Raman Paranjape1 \\ ${ }^{1}$ Electronic Systems Program, Faculty of Engineering, University of Regina, Regina, Canada \\ ${ }^{2}$ Industrial Systems Program, Faculty of Engineering, University of Regina, Regina, Canada \\ Email: Erui198311@gmail.com, Mehran.Mehrandezh@uregina.ca, Raman.Paranjape@uregina.ca
}

Received 27 September 2014; revised 20 October 2014; accepted 10 November 2014

Academic Editor: Wahied Gharieb Ali, Computers \& Systems Engineering Department, Faculty of Engineering, Ain Shams University, Egypt

Copyright (C) 2014 by authors and Scientific Research Publishing Inc.

This work is licensed under the Creative Commons Attribution International License (CC BY). http://creativecommons.org/licenses/by/4.0/

(c) (i) Open Access

\section{Abstract}

The sense of touch as a man-machine communication channel can be as acute as the sense of sight and sound. In some scenarios such as those seen in aerobatics, stunt flying, and combat flights, tactile sensors can even outperform the conventional non-contact sensors in terms of situation awareness. Fusion of tactile sensory information with those obtained via sight and sound can avoid diverting the user's attention away from the operational task at hand as well. In this study, the performance of an operator, to servo control the motion of a 2-dof model helicopter with pitch/yaw maneuverability, subjected to an intuitive body-referenced arrangement of a cluster of vibro-tactile sensors is investigated. A blindfolded operator will then control the helicopter to a safe attraction zone via a joystick based on this tactile sensory information. A fine-tuned local controller would take over for the end-of-motion precise homing. This study can pave the way towards a systematic integration and characterization of tactile sensors in high performance weapon platforms with improved situation awareness in visually awkward maneuvers such as those seen in aerial combat scenarios.

\section{Keywords}

Haptics, Flight Control, Linear Quadratic Regulators (LQR), Tactile Immersive Control, 2-Dof Model Helicopter, Mechatronics, Real-Time Control

\section{Introduction}

Highly maneuverable flying machines such as helicopters are widely used in search and rescue, and surveillance due to their agility [1]. Control of these machines lends itself as a challenging Multi-Input Multi-Output (MIMO) 
problem due to the nonlinear dynamics and strong interaction between controlled variables. Incorporating haptic sensors into the closed-loop feedback control of flying machines has recently drawn a great deal of attention in both academia and the industry sector [2]. When employing body-referenced haptic sensors, the man-machine interaction can be as acute as that when using sense of sight and/or sound. In [3], a haptic interface for UAV collision avoidance was used. It was reported that, with the limited visual information, the collision could be avoided using haptic clues. Literature pertinent to the control of highly maneuverable aircrafts is vast. In particular, the robust and optimal controls of helicopters have been studied for the past two decades, (e.g., Refs. [1] [4]).

An effective human-in-the-loop hybrid control strategy based on sensory information obtained via an array of body-mounted haptic sensors (i.e., vibrating motors) is proposed. This method was successfully tested on a 2dof model helicopter. The control strategy is summarized below.

At the first stage, a user attempts to navigate the model helicopter to an attraction zone through haptic sensory feedback by using a joystick. In the second stage, an autonomous nonlinear controller takes over making the helicopter hover at steady state with high precision. A simple switching algorithm is adopted for smooth transition between the human- and computer-based controls.

The experimental model helicopter used in this study has two degrees of freedom, namely pitch and yaw (see Figure 1). The pitch propeller is driven by a voltage-controlled DC motor. Nose up is considered as positive pitch. Correspondingly, the yaw propeller is driven by a smaller voltage-controlled DC motor. Due to the gyroscopic effect, the propeller causing pitch would also cause a load torque on the motor's shaft which is in turn seen at the yaw axis. The model helicopter is pivoted on a fixed stand. Its center of gravity is located off the pivot point with a considerable offset in a way that the gravitation force will push the helicopter's nose down all the time. Pitch and yaw angles are measured through optical encoders. The pitch angle is limited to \pm 40 degrees from the level configuration, while, thanks to the slip-ring employed at the pivot point, the yaw angle can vary, and being measured by an optical encoder, indefinitely.

The layout of the paper is as follows: The governing dynamics equations of the system are described in Section 2. The control strategy is explained in Section 3. The experimental setup and results are given in Sections 4 and 5 , respectively. Section 6 concludes the paper and described the future works.

\section{Dynamics Model of the Helicopter}

Figure 1 shows a schematic of the main components of the model helicopter. The governing dynamics equations are as follows [5]:

$$
\begin{gathered}
\left(J_{e q, p}+m_{\mathrm{heli}} l_{\mathrm{cm}}^{2}\right) \ddot{\theta}=K_{p p} V_{m, p}+K_{p y} V_{m, y}-m_{\mathrm{heli}} g l_{c m} \cos (\theta)-B_{p} \dot{\theta}-m_{\mathrm{heli}} l_{\mathrm{cm}}^{2} \sin (\theta) \cos (\theta) \dot{\psi}^{2} \\
\left(J_{e q, y}+m_{\mathrm{heli}} l_{\mathrm{cm}}^{2} \cos ^{2}(\theta)\right) \ddot{\psi}=K_{y y} V_{m, y}+K_{y p} V_{m, p}-B_{y} \dot{\psi}+2 m_{\mathrm{heli}} l_{\mathrm{cm}}^{2} \sin (\theta) \cos (\theta) \dot{\psi} \dot{\theta}
\end{gathered}
$$

Definition of parameters in Equations (1) and (2) are given in Table 1.

The nonlinear governing dynamics given in Equations (1) and (2) can be linearized about the quiescent point defined at $\theta_{0}=0^{\circ}, \psi_{0}=0^{\circ}$, and $\dot{\theta}_{0}=\dot{\psi}_{0}=0^{\circ}$ as follows:

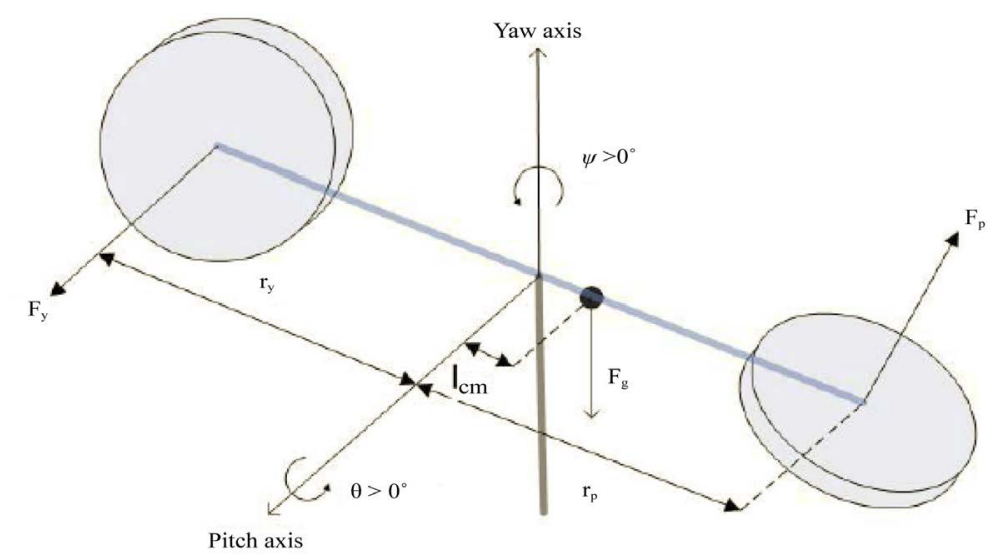

Figure 1. A schematic of the 2-dof model helicopter. 
Table 1. Physical parameters of the system.

\begin{tabular}{cccc}
\hline Symbol & Definition & Value & Unit \\
\hline$J_{e q, p}$ & Moment of inertia (pitch direction) & 0.0384 & $\mathrm{~kg} \cdot \mathrm{m}^{2}$ \\
$J_{e q, y}$ & Moment of inertia (yaw direction) & 0.0432 & $\mathrm{~kg} \cdot \mathrm{m}^{2}$ \\
$m_{\text {heli }}$ & Mass of the helicopter & 1.3872 & $\mathrm{~kg}$ \\
$l_{\mathrm{cm}}$ & Distance from center of mass to yaw axis & 0.186 & $\mathrm{~cm}$ \\
$K_{p p}$ & Thrust torque constant acting on pitch axis from pitch motor & 0.204 & $\mathrm{~N} \cdot \mathrm{m} / \mathrm{V}$ \\
$K_{y y}$ & Thrust torque constant acting on yaw axis from yaw motor & 0.072 & $\mathrm{~N} \cdot \mathrm{m} / \mathrm{V}$ \\
$K_{p y}$ & Thrust torque constant acting on pitch axis from yaw motor & 0.0068 & $\mathrm{~N} \cdot \mathrm{m} / \mathrm{V}$ \\
$K_{y p}$ & Thrust torque constant acting on yaw axis from pitch motor & 0.0219 & $\mathrm{~N} \cdot \mathrm{m} / \mathrm{V}$ \\
$V_{m, p}$ & Power voltage acting on the pitch motor & Variable & $\mathrm{V}$ \\
$V_{m, y}$ & Power voltage acting on the yaw motor & Variable & $\mathrm{V}$ \\
\hline
\end{tabular}

$$
\begin{gathered}
\left(J_{e q, p}+m_{\mathrm{heli}} l_{\mathrm{cm}}^{2}\right) \ddot{\theta}=K_{p p}\left(V_{m, p}-\frac{m_{\mathrm{heli}} g l_{\mathrm{cm}}}{K_{p p}}\right)+K_{p y} V_{m, y}-B_{p} \dot{\theta} \\
\left(J_{e q, y}+m_{\mathrm{heli}} l_{\mathrm{cm}}^{2}\right) \ddot{\psi}=K_{y y} V_{m, y}+K_{y p} V_{m, p}-B_{y} \dot{\psi}
\end{gathered}
$$

The linearized dynamics equations can be presented in the state space, disregarding the gravitational torque term, namely $m_{\text {heli }} g l_{\mathrm{cm}}$ as follows:

$$
\dot{\boldsymbol{X}}=\left[\begin{array}{cccc}
0 & 0 & 1 & 0 \\
0 & 0 & 0 & 1 \\
0 & 0 & \frac{-B_{p}}{J_{e q, p}+m_{\mathrm{heli}} l_{\mathrm{cm}}^{2}} & 0 \\
0 & 0 & 0 & \frac{-B_{p}}{J_{e q, p}+m_{\mathrm{heli}} l_{\mathrm{cm}}^{2}}
\end{array}\right] \boldsymbol{X}+\left[\begin{array}{cc}
0 & 0 \\
0 & 0 \\
\frac{K_{p p}}{J_{e q, p}+m_{\mathrm{heli}} l_{\mathrm{cm}}^{2}} & \frac{K_{p y}}{J_{e q, p}+m_{\mathrm{heli}} l_{\mathrm{cm}}^{2}} \\
\frac{K_{y p}}{J_{e q, p}+m_{\mathrm{heli}} l_{\mathrm{cm}}^{2}} & \frac{K_{y y}}{J_{e q, p}+m_{\mathrm{heli}} l_{\mathrm{cm}}^{2}}
\end{array}\right] \boldsymbol{u}
$$

where the state and control vectors are: $\boldsymbol{X}=[\theta, \psi, \dot{\theta}, \dot{\psi}]^{\mathrm{T}}$ and $\boldsymbol{u}=\left[V_{m, p}, V_{m, y}\right]^{\mathrm{T}}$ respectively.

As can be seen from Equation (1), the gravitational force generates a torque around the pivot point as $\tau_{g}=m_{\text {heli }} g l_{\mathrm{cm}} \cos (\theta)$. In order to compensate this torque, a non-linear term, $u_{f f}$ was fed-forward to the voltage provided to the pitch DC motor as follows:

$$
V_{p}=V_{m, p}+u_{f f}
$$

where $u_{f f}$ is:

$$
u_{f f}=K_{f f} \frac{m_{\text {heli }} g l_{\mathrm{cm}} \cos \left(\theta_{d}\right)}{K_{p p}}
$$

In Equation (7), $K_{f f}$ denotes the feed-forward control gain (normally considered as 1 ) and $\theta_{d}$ denotes the desired pitch angle. The gravitational force would have no effect on yaw motion. Therefore, the overall pitch/yaw control input denoted by $\left[V_{p}, V_{y}\right]^{\mathrm{T}}$ can be presented as:

$$
\left\{\begin{array}{l}
V_{p}=V_{m, p}+u_{f f} \\
V_{y}=V_{m, y}
\end{array}\right.
$$

\section{The Proposed Control Strategy}

The control strategy is carried out in two phases. In the first phase, a human operator attempts to bring the robot 
within an attraction zone using a joystick via haptic sensory information. We call this a human-in-the-loop control strategy. In the second phase, a nonlinear controller takes over for the precise end-of-motion servoing purpose. A simple switching law is adopted for smooth transition between the two controllers. The control strategies and the switching law are described in the following sections.

\subsection{Human-in-the-Loop Control}

The key to the successful integration of vibro-tactile sensory information into a control loop is to convey an organized tactile sensation to the user via lightweight and compact devices mounted on user's body without impairing his/her movement. In this context, an array of four small-size vibrating DC motors (denoted as front, back, left and right motors) was mounted on the user's body (see Figure 2). The voltage provided to the quadruplet vibro-motors, and correspondingly their vibration amplitudes, will change proportional to the error and also rate of change of error between the pitch and yaw angles and their desired values. Mathematically this can be written as:

$$
\left[u_{p}, u_{m}\right]^{\mathrm{T}}=\left[u_{\mathrm{ff}}, 0\right]^{\mathrm{T}}-K_{\text {calib }} K_{\mathrm{LQR}}\left(\boldsymbol{X}-\boldsymbol{X}_{d}\right)
$$

where $u_{p}$ and $u_{m}$ denote the voltages provided to the two pairs (i.e., front-back and left-right) of the bodymounted vibro-motors corresponding to pitch and yaw errors, respectively, the $K_{\text {calib }}$ denotes the calibration constant, the $K_{\mathrm{LQR}}$ denotes the Linear Quadratic Regulator (LQR) gain, and $\boldsymbol{X}_{d}$ denotes the desired state of the helicopter. The arrangement of the voltages provided to the quadruplet vibro motors are as follows:

$$
V_{F}=\left\{\begin{array}{ll}
u_{p} & \text { if } u_{p} \geq 0 \\
0 & \text { otherwise }
\end{array}, \quad V_{B}=\left\{\begin{array}{ll}
-u_{p} & \text { if } u_{p} \leq 0 \\
0 & \text { otherwise }
\end{array}, \quad V_{R}=\left\{\begin{array}{ll}
u_{y} & \text { if } u_{y} \geq 0 \\
0 & \text { otherwise }
\end{array}, \quad V_{L}=\left\{\begin{array}{ll}
-u_{y} & \text { if } u_{y} \leq 0 \\
0 & \text { otherwise }
\end{array} .\right.\right.\right.\right.
$$

In Equation (10), $V_{F}, V_{B}, V_{R}$, and $V_{L}$ denote the voltages provided to the front, back, right and left motors mounted on operator's body as seen in Figure 2. In the next section, a description on calculating the $K_{\mathrm{LQR}}$ is given.

\subsubsection{The Linear Quadratic Regulator, an Overview}

In a Linear Quadratic Regulator (LQR), the control gain, $K$ in a linear full-state feedback control law of the form $u=-K \boldsymbol{X}$ is calculated by minimizing a quadratic objective function, $J$ defined as:

$$
J=\int_{0}^{\infty}\left(\boldsymbol{X}^{\mathrm{T}} Q \boldsymbol{X}+\boldsymbol{u}^{\mathrm{T}} R \boldsymbol{u}\right) \mathrm{d} t
$$

where $Q$ and $R$ are weighting matrices on states and the control variables, respectively. The following weighting matrices were adopted in our experiments:

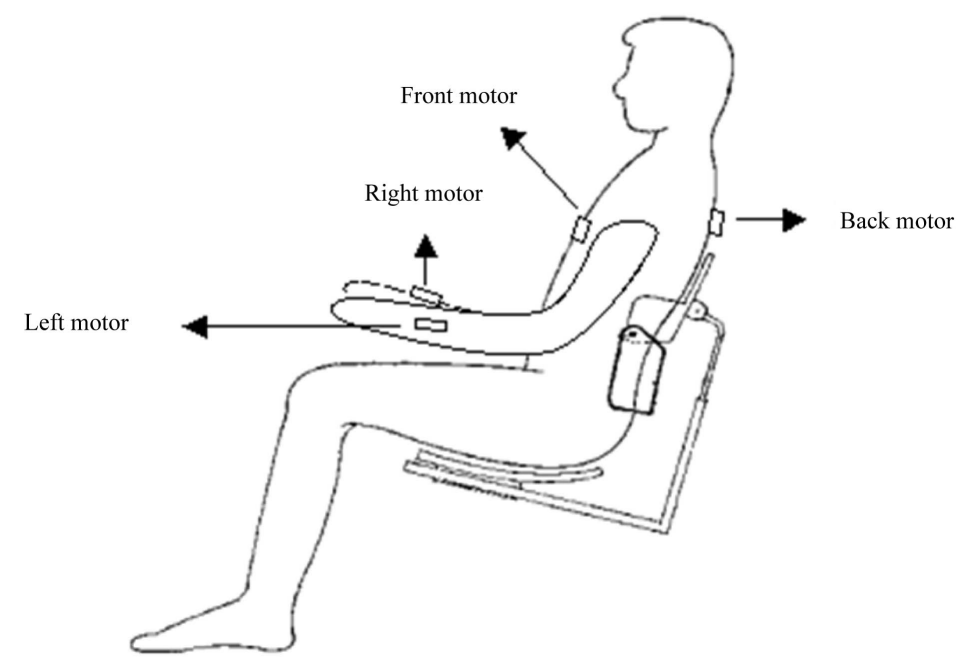

Figure 2. Four vibro-tactile sensors mounted on human's body. 


$$
Q=\left[\begin{array}{cccc}
200 & 0 & 0 & 0 \\
0 & 200 & 0 & 0 \\
0 & 0 & 100 & 0 \\
0 & 0 & 0 & 100
\end{array}\right], \quad \text { and } \quad R=\left[\begin{array}{ll}
1 & 0 \\
0 & 1
\end{array}\right]
$$

The LQR control gain matrix, $K_{\mathrm{LQR}}$ was calculated as:

$$
K_{\mathrm{LQR}}=\left[\begin{array}{cccc}
14.1 & 1.33 & 7.33 & 0.95 \\
-1.33 & 14.1 & -0.26 & 7.99
\end{array}\right]
$$

\subsubsection{The Switching Law}

The user controls the motion of the model helicopter based on vibro-tactile sensation he/she receives through four body-mounted motors. A human-based control would shift to an autonomous control strategy after helicopter is brought to a safe zone (i.e., attraction zone). A weighted 2-norm of the state error as a performance index, $\eta$ was used to trigger the switching between the human- and computer-based controllers. Mathematically it can be represented as:

$$
\eta=\sum_{i=1}^{4} w_{i}\left\|x_{i}-x_{i d}\right\|
$$

where $w_{i}$ denotes the weighting factors, $x_{i}$ denote the states, and $x_{i d}$ denotes the desired value of states.

Weighting factors, $w_{i}$ and the calibration constant, $K_{\text {calib }}$ were tuned offline during operator's training based on his/her level of comfort when controlling the model helicopter. The overall structure of the proposed control strategy is depicted in Figure 3.

\subsection{End-of-Motion Servo Control}

A full-state feedback control law similar to that in LQR was developed for the end-of-motion servoing. However, a loop-shaping optimization process was utilized to calculate the controller gains. The nonlinear model of the helicopter was assumed within the optimization process. Furthermore, the pitch/yaw motor saturation was taken into consideration through the optimization process as well. Geometric hard constrains on parameters such as settling time, rise time and the overshoot in system's response were defined offline prior to optimization. The Response Optimization Toolbox from Math Work was used for this purpose [6]. As an example, Figure 4 shows the simulated yaw response of the model helicopter when using the LQR design described in Section (3.1.1). Figure 5 shows the system's response based on the response optimization technique described earlier. As can be seen from Figure 5, a lesser overshoot, settling time, and rise time were achieved without saturating the yaw motor. Figure 6 shows the geometric constrains imposed on the system's response along with a number of iterations on calculated response trajectories. It is noteworthy that two integral terms on pitch and yaw angles were also added to the state vector further on to reduce the steady state error. The modified state vector $\tilde{X}$ was:

$$
\tilde{X}=\left[X, \int \theta \mathrm{d} t, \int \psi \mathrm{d} t\right]^{\mathrm{T}}
$$

The optimal gain $K_{\mathrm{opt}}$ was calculated as:

$$
K_{\text {opt }}=\left[\begin{array}{cccccc}
19.02 & 2.13 & 7.37 & 1.36 & 6.96 & 0.80 \\
-1.63 & 20.77 & -1.22 & 11.07 & -1.50 & 7.00
\end{array}\right]
$$

\section{Experimental Setup}

Figure 7 shows the experimental apparatus. Two power modules were used to drive pitch and yaw motors of the model helicopter. Linear Pulse Width Modulated (PWM) servo amplifiers were also used to drive body-mounted vibrating motors. The motion of the helicopter can be controlled in real time via a Logitech joystick (shown in the figure) or completely autonomously. The Q8 board from Quanser was used for control and data acquisition in real time [7]. Wincon from Quanser along with a third-party real-time kernel provide a real-time operating environment within the Simulink. The Q8 board can support 8 analogue outputs and 8 quadratic encoder chan- 


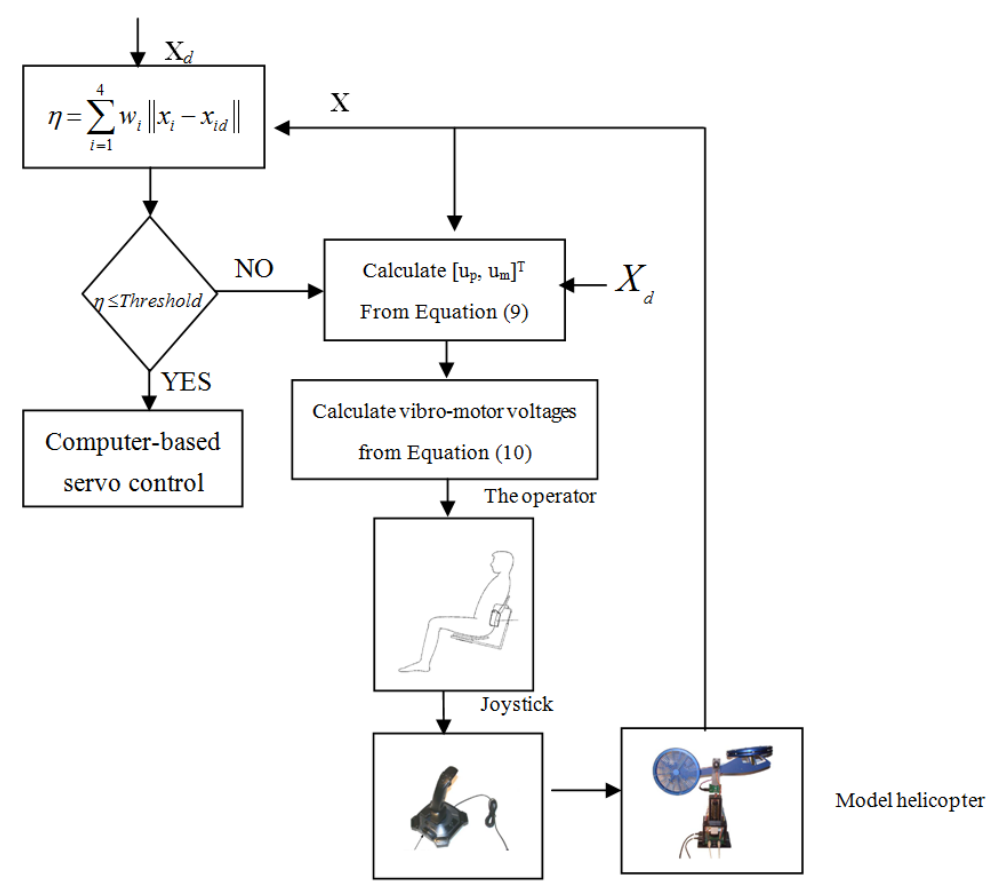

Figure 3. The proposed control structure.

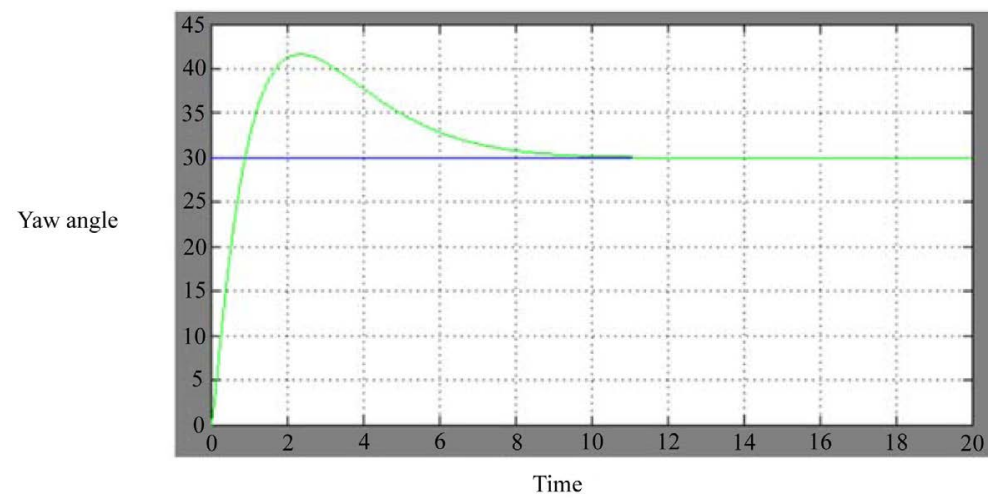

Figure 4. Simulated yaw response of the 2-dof model helicopter via LQR + Integrator.

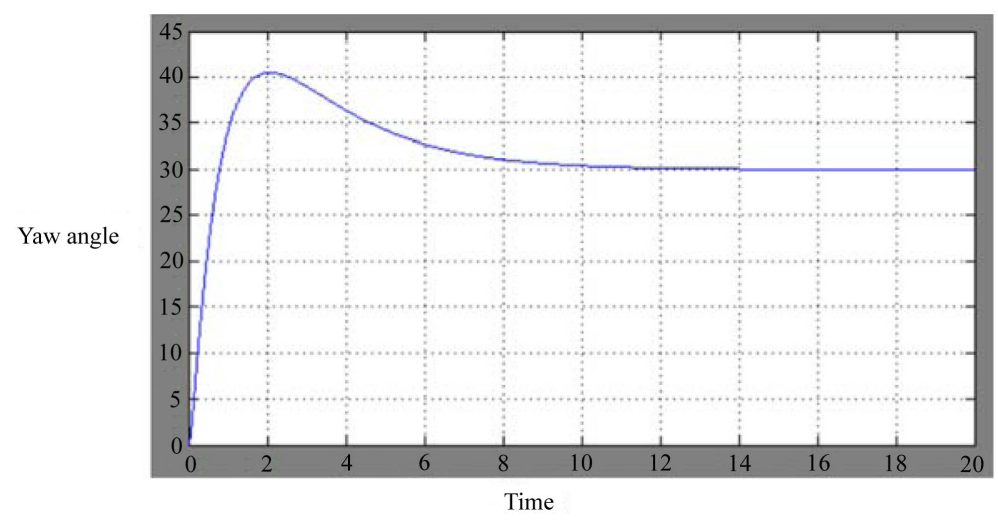

Figure 5. Simulated yaw response of the 2-dof model helicopter via loopshaping and response optimization. 


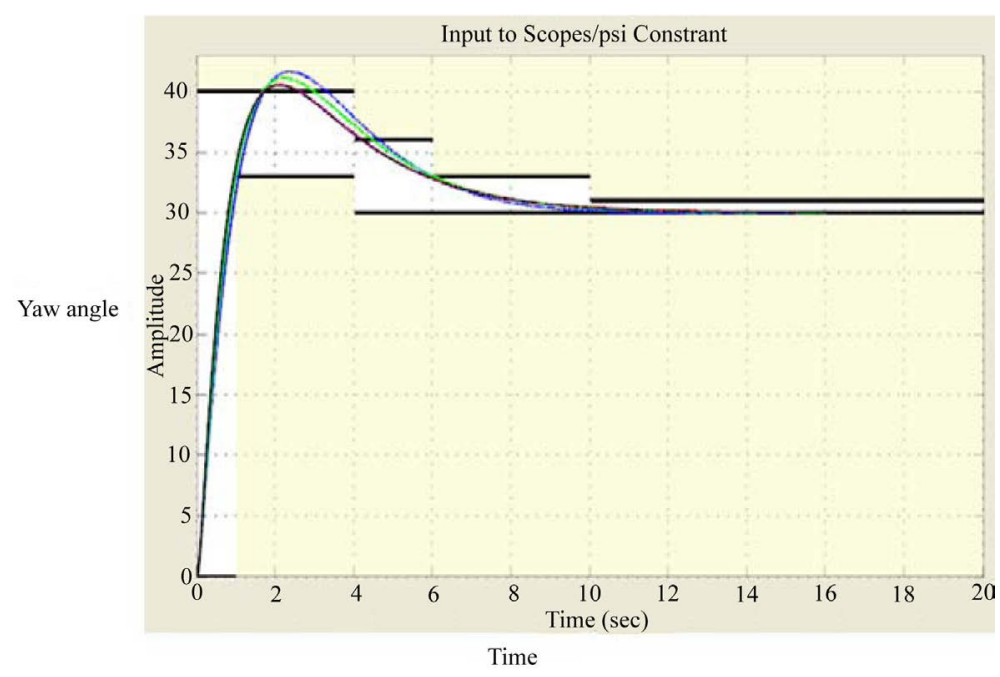

Figure 6. User-defined geometric hard constraints on the 2-dof model helicopter's yaw response.

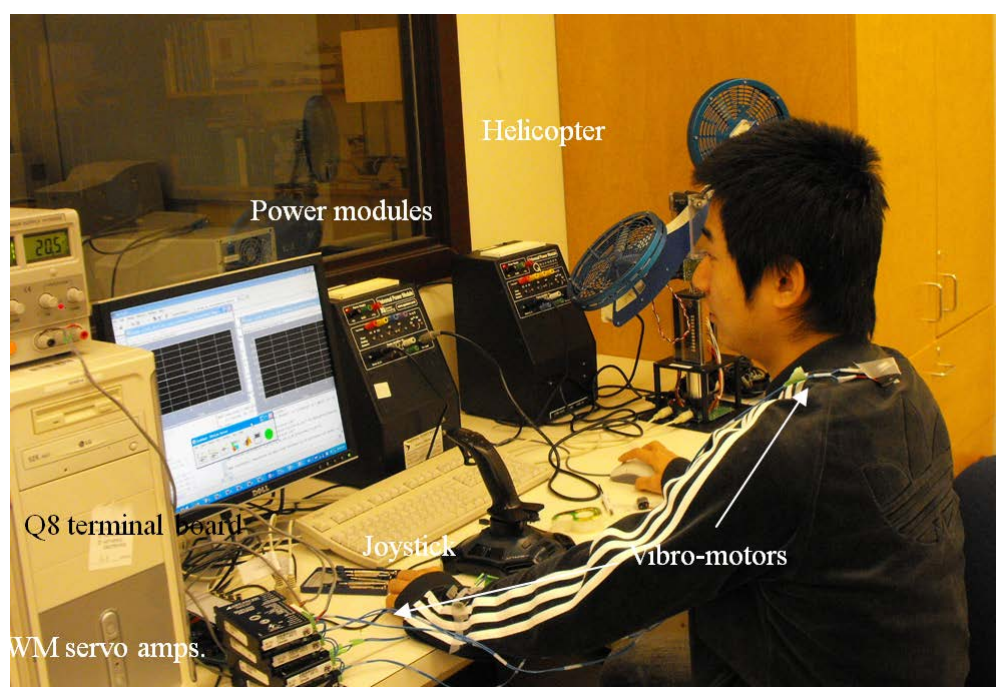

Figure 7. The experimental apparatus.

nels. Two rotary optical encoders measure the pitch and yaw angles in real time. Pitch motion is limited to \pm 40 degrees from the level configuration. However, the yaw angle can change indefinitely thanks to the slip-ring mechanism used at the pivot point connecting the stand and the helicopter's main body. The pitch motor can generate a larger thrust than that in the yaw motor. This extra power would be needed to compensate for the gravitational torque around the pivot due to the offset between the helicopter's center of gravity and the hinge on the helicopter's stand.

\section{Experimental Result}

Figure 8 and Figure 9 show two representative experimental results. The desired pitch and yaw angles were set at 0 and 90 degrees, respectively in both experiments. Pitch and yaw angles along with voltages provided to the pitch/yaw motors are shown in figures. The initial elapsed time for controllers to take effect in both experiments was set at 3 seconds approximately. In the first experiment shown in Figure 8, the human-in-the-loop control strategy is in place for 15 seconds following the initialization. The computer-based controller starts at 18 seconds introducing some small overshoots in both pitch and yaw motions before bringing the helicopter to steady state. 


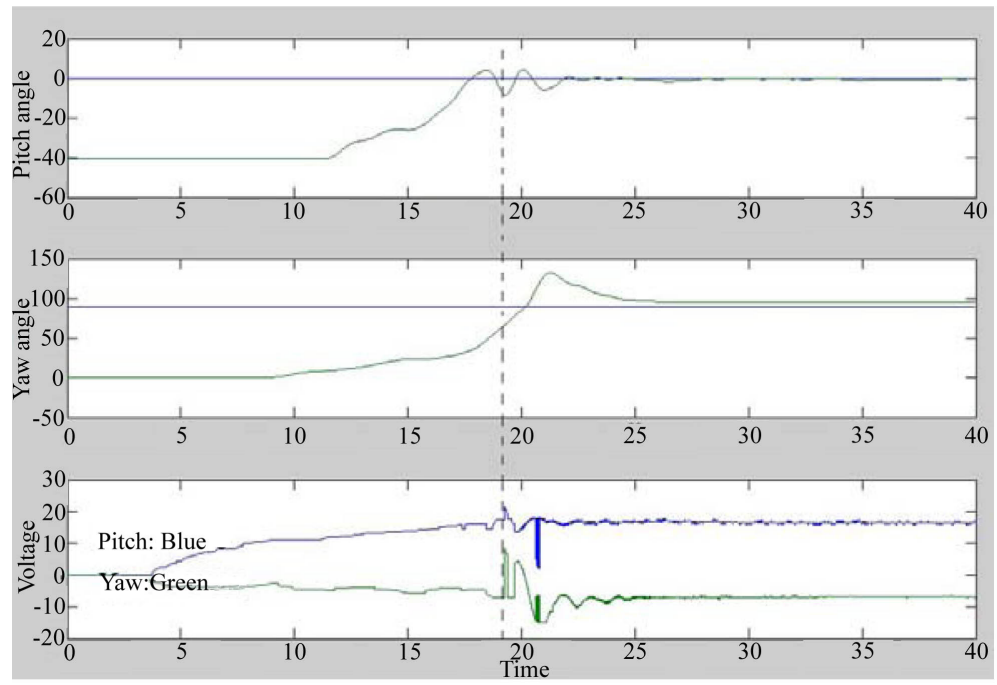

Figure 8. Experimental results \#1 (original in color).

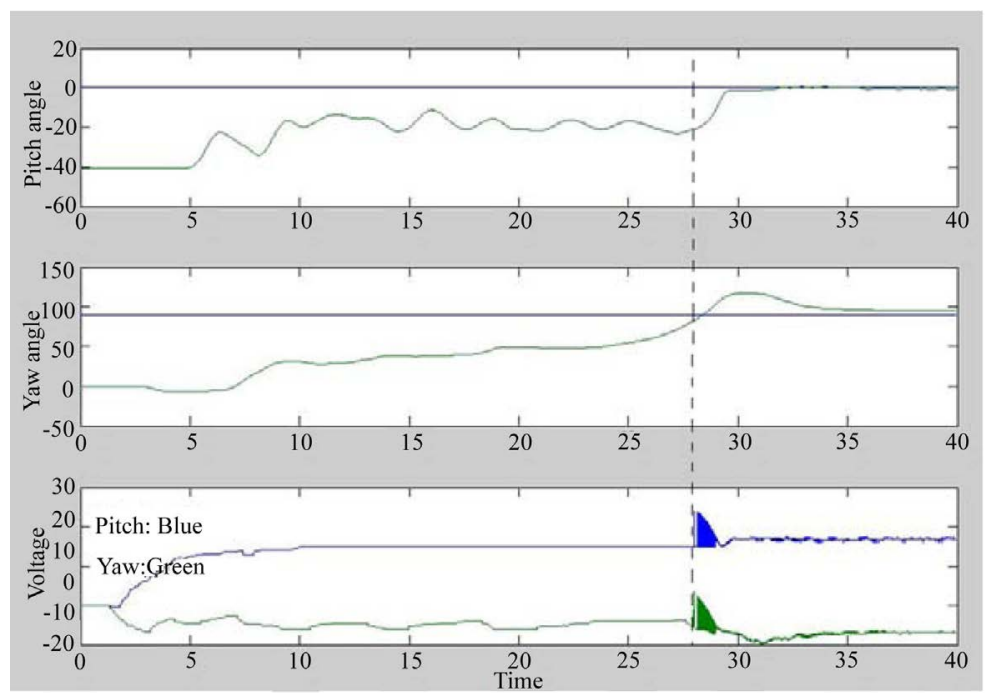

Figure 9. Experimental result after changing parameters (original in color).

In the second experimentation, the calibration constant, $K_{\text {calib }}$, and also the switching threshold, $\eta$ were changed based on operator's learning and comfort level. A lesser overshoot was achieved but at the cost of an increased settling time. It is noteworthy that the sensitivity of the joystick was also decreased in the second experimentation. In both experiments the operator could guide the model helicopter to a safe zone blindfolded. In both figures, the dashed line represents the time at which the computer-based controller takes effect.

\section{Conclusions and Future Work}

A methodology to incorporate tactile sensory information into the feedback control loop of a 2-dof model helicopter was addressed. It was shown through some experiments that body-referenced touch sensors can provide the human operator with sufficient information to guide a free-flying object even in the case that visual clues are obstructed. A blindfolded operator, after going through a training period, could navigate the model helicopter to a safe zone. The overall control strategy was divided into two main stages, namely the human-in-the-loop and the computer-based control. An LQR-based control strategy would suffice to generate proper control cues to the operator, subjected to a cluster of vibro-tactile motors mounted on his/her body. A computer-based control takes effect at the end of motion for precise homing. 
Further characterization of the human learning curve for the more robust and smooth control of our model helicopter is under investigation. Incorporating visual clues when controlling the model helicopter in awkward maneuvers such as those found in stunt flying in form of a sensor fusion technique will be also investigated. Incorporating collision avoidance strategies into the control loop through tactile sensory information on a free flying UAV is envisioned in a long term.

\section{References}

[1] McLean, D. (2006) Automatic Flight Control Systems. Measurement and Control, 36, 172-177. http://dx.doi.org/10.1177/002029400303600602

[2] Yokokohji, Y., Tubouchi, T., Tanaka, A. and Yoshida, T. (2006) Guidelines for Human Interface Design of Rescue Robots. Proceedings of the SICE-ICASE IEEE International Conference, Busan, 18-21 October 2006, 3455-3460.

[3] Lam, T.M., Mulder, M. and Paassen, V. (2007) Haptic Interface for UAV Collision Avoidance. International Journal of Aviation Psychology, 17, 167-195. http://dx.doi.org/10.1080/10508410701328649

[4] Gwo-Ruey, Y. (2007) Robust-Optimal Control of a Nonlinear Two Degree-of-Freedom Helicopter. Proceedings of the 6th IEEE/ACIS International Conference on Computer and Information Science, Melbourne, 11-13 July 2007, 744-749.

[5] Quanser Company (2014) Quanser 2 DOF Helicopter User Manual. http://users.encs.concordia.ca/ realtime/elec483/docs/2\%20DOF\%20Helicopter\%20User\%20Manual.pdf

[6] Mathwork (2014) Simulink Response Optimization User’s Guide. http://www.mathworks.com/help/sldo/index.html\#response-optimization

[7] Quanser (2014) Q8 WinCon Support \& Installation Guide. http://www.quanser.com/Products/q8 
Scientific Research Publishing (SCIRP) is one of the largest Open Access journal publishers. It is currently publishing more than 200 open access, online, peer-reviewed journals covering a wide range of academic disciplines. SCIRP serves the worldwide academic communities and contributes to the progress and application of science with its publication.

Other selected journals from SCIRP are listed as below. Submit your manuscript to us via either submit@scirp.org or Online Submission Portal.
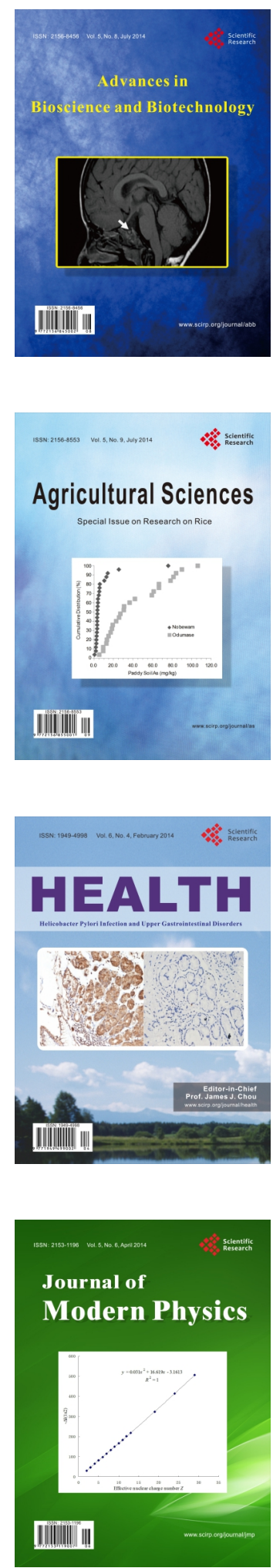
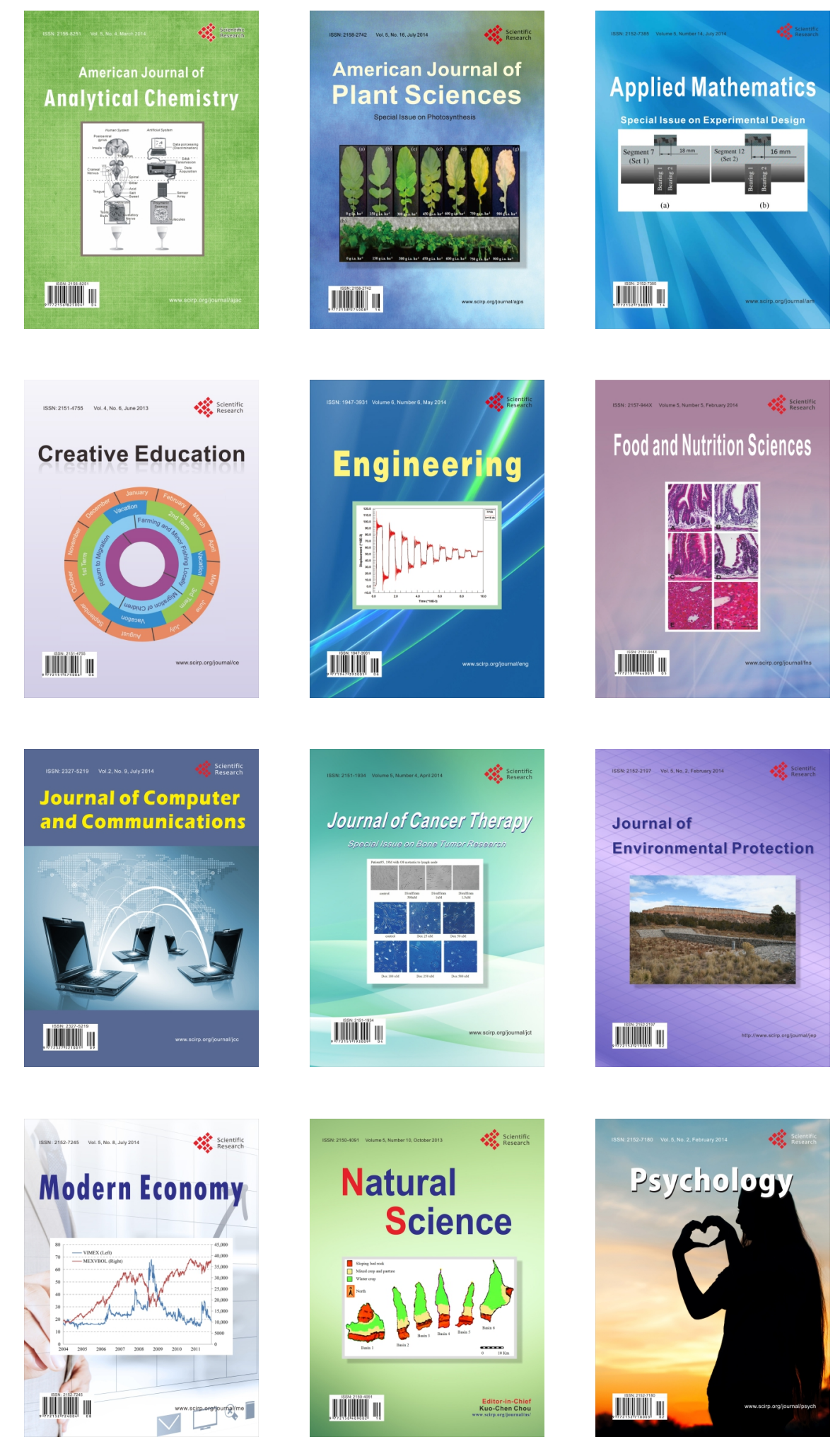